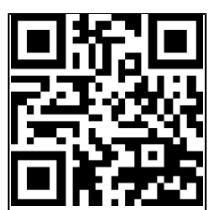

Editor's choice Scan to access mo free content

\title{
Summer heat: a cross-sectional analysis of seasonal differences in sexual behaviour and sexually transmissible diseases in Melbourne, Australia
}

\author{
Vincent J Cornelisse, ${ }^{1,2}$ Eric P F Chow, ${ }^{1,2}$ Marcus Y Chen, ${ }^{1,2}$ Catriona S Bradshaw, $^{1,2}$ \\ Christopher K Fairley ${ }^{1,2}$
}

- Additional material is published online only. To view please visit the journal online (http://dx.doi.org/10.1136/ sextrans-2015-052225).

${ }^{1}$ Central Clinical School, Faculty of Medicine, Nursing and Health Sciences, Monash University, Melbourne, Victoria, Australia

${ }^{2}$ Melbourne Sexual Health Centre, Alfred Health, Carlton, Victoria, Australia

\section{Correspondence to} Dr Vincent J Cornelisse, Melbourne Sexual Health Centre, Alfred Health, 580 Swanston St, Carlton, VIC 3052, Australia; vcornelisse@mshc.org.au; echow@mshc.org.au

Received 29 June 2015 Revised 12 October 2015 Accepted 17 October 2015 Published Online First 6 November 2015
ABSTRACT

Objectives To date, no study has correlated seasonal differences in sexual behaviour with the seasonal differences in sexually transmitted infections (STIS); and no seasonal study of STIs has been conducted in the southern hemisphere. Our study aimed to describe seasonal differences in sexual behaviour and correlate this with seasonal differences in STI diagnoses in Melbourne, Australia.

Method This was a cross-sectional study of individuals attending the Melbourne Sexual Health Centre over a 9year period from 2006 to 2014. We conducted separate analyses for men who have sex with men (MSM) and men who have sex with women (MSW), and women. Seasonal patterns of sexual behaviour and STI positivity were examined within each group.

Results All groups reported a higher number of partners over the preceding three months for consultations in summer compared with winter (MSM mean 5.48 vs 5.03 ; MSW mean 2.46 vs 2.31; women mean 1.83 vs 1.72). Urethral gonorrhoea diagnoses among MSM were higher in summer compared with winter (OR 1.23, 95\% Cl 1.04 to 1.46). Similarly, nongonococcal urethritis (NGU) diagnoses among MSW were the highest in summer (OR 1.11,95\% Cl 1.03 to 1.20), but there was no seasonal difference in NGU diagnoses when we adjusted for partner numbers. In women, pelvic inflammatory disease (PID) diagnoses peaked in autumn, when rates were higher than in winter (OR 1.30, 95\% Cl 1.09 to 1.55).

Conclusions Our results describe a peak in sexual partner number and STI diagnoses during consultations in summer in men and a rise in PID in autumn in women.

\section{INTRODUCTION}

If there is a seasonal difference in rates of sexually transmitted infections (STIs), then public health campaigns could be most effective if they were timed accordingly. Previous studies in the USA have shown a peak in rates of STIs in summer and early autumn. ${ }^{1-3}$ Two studies in the $\mathrm{UK}^{4}{ }^{5}$ have shown a two-peak seasonal distribution in STI incidence, with peaks in both the first quarter and third quarter of the year, corresponding to the time after Christmas holidays and summer holidays, respectively. A study of gonorrhoea and urethritis rates in Saharan Africa ${ }^{6}$ also reported a seasonal difference, with highest rates from January to May, coinciding with the postharvest season during which young adults migrate for work, and perhaps is a parallel for the 'summer break' period in the USA. None of these studies correlated the seasonal difference in STI rates with seasonal differences in behavioural risk factors for STI such as partner numbers and condom use.

Other studies have assessed seasonal differences in sexual behaviours without assessing STI rates. A study conducted in the USA observed a peak of first sexual intercourse or 'loss of virginity' in June through to August; this seasonal difference was more pronounced in school-aged teenagers than in older youths, suggesting that school holidays may be an influence. ${ }^{7}$ A small prospective cohort study of sexual behaviour amongst women in the USA recorded increased sexual activity in summer, but no increase in number of partners. Condoms were used more consistently in summer than in winter. A study in the $\mathrm{UK}^{5}$ reported an increase in pregnancy terminations in the first quarter of the year, corresponding to what are presumed to be unintended conceptions around the Christmas period. Correspondingly, they showed peaks in condoms sales over Christmas and over summer.

To date, no study has compared seasonal differences in sexual behaviour and other STI risks with seasonal differences in STI positivity; and no seasonal study has been conducted in the southern hemisphere. The aim of this study was to assess and correlate these seasonal differences in order to inform the design and timing of public health campaigns targeted to reduce STI rates in Australia.

\section{METHODS}

This was a cross-sectional study of all individuals attending the Melbourne Sexual Health Centre (MSHC) over a 9-year period from 1 January 2006 to 31 December 2014 inclusive. As of 2013, the MSHC provided approximately 35000 sexual health consultations annually, about $37 \%$ of these for men who have sex with men (MSM). ${ }^{9}$ It is a free walk-in service; no referrals are required. Prior to seeing the triage nurse, attendees complete a computer-assisted self-interview (CASI) that collects their demographic details and history of sexual behaviours and drug use. ${ }^{10}$ On most days, a small number of patients are triaged out of the service due to the service operating at full capacity. The triage nurse makes the triaging decision after patients have completed their CASI questionnaire. 
For the purposes of this study, MSM were defined as those men who reported at least one male sexual partner in the last three months irrespective of female partners. Heterosexual men (MSW) were defined as those who reported female but no male sexual partners in the last three months. We included women who reported at least one male partner in the last three months and who were currently not engaged in sex work. Transgender people were excluded. Consultations with incomplete behavioural data were excluded.

Behavioural data and STI diagnoses from each patient were stratified by the season that corresponded to the date of their consultation at the MSHC.

For MSM and heterosexual men, we assessed the proportion of consultations with urethral gonorrhoea, symptomatic nongonococcal urethritis (NGU) or first episode of genital herpes simplex virus (HSV). For women, we assessed the proportion of consultations with HSV or pelvic inflammatory disease (PID). Conditions with short incubation periods and high symptomatic rates were specifically chosen so their presentation at the clinic would be indicative of disease incidence and recent risk practices, and hence would allow for assessment of seasonality. To confirm that our selection of STIs for seasonal analysis was appropriate, we also examined other common STIs, including genital warts, syphilis, chlamydia and mycoplasma genitalium infections. None of these showed a seasonal difference.

Differences in behavioural data were examined by season, including condom use, injecting drug use (IDU) and the number of sexual partners reported for the three months preceding the consultation date. Behavioural data were extracted from the electronic health record, in which entries were derived from CASI entries and were reviewed by the attending clinician. Condom use data for anal or vaginal sex in the past three months were stratified into two groups (consistent use of condoms vs inconsistent or no condom use). IDU data in the past three months were stratified into two groups (yes vs no). Some individuals in the data set reported extremely large numbers of sexual partners over the last three months (up to 600 for MSM, and 100 for heterosexual men and women). In order to prevent skewing of the data analysis by these outliers, and to allow us to perform multinomial regression analyses, all individually reported partner numbers over the last three months were recoded into four categories: (1) 1-2 partners; (2) 3-4 partners; (3) 5-6 partners and (4) $\geq 7$ partners. These categories were chosen as they represent a reasonable spread of partner numbers around the median partner number for each population group $(\mathrm{MSM}=3 ; \mathrm{MSW}=2$; women=1).

The data were analysed using SPSS V.21. Descriptive statistics such as the mean number of partners for each population by season were calculated from the raw data. Univariate and multivariate logistic regressions were performed to examine the association between seasons and STI diagnoses. Multivariate logistic regression was conducted by adjusting for a range of risk factors such as partner number, condom use, IDU, age and year of consultation. Multinomial logistic regression was performed to examine association between partner number categories and seasons. Adjusted ORs (aORs) and 95\% CIs were calculated.

We repeated our analysis of seasonal differences in sexual behaviours with the inclusion of those patients who were triaged out of the service to assess whether our primary analysis was subject to a bias as a result of the triage process.

For the purpose of providing a more detailed illustration of the trend in partner number and STI diagnoses, we graphically displayed centred 30-day moving averages for each of the population subgroups (figure 1). To minimise the effect of outliers on this graph, we restricted individually reported partner numbers to the 95th percentile for each population group; those individuals who reported partner numbers greater than the 95th percentile for their population had their partner number reduced to the 95th percentile (ie, MSM >20 partners, MSW >6 partners and for women $>4$ partners over a 3 -month period).

In figure 1, STI diagnoses for any particular day on the graph is the percentage of individuals on that day who were diagnosed with that STI. A 30-day moving average was chosen because a shorter time period resulted in excessive day-to-day variability. A graph of the raw data is included in the online supplementary figure S1.

\section{RESULTS}

The total number of consultations in each season for each population (MSM, MSW, women) did not vary substantially between seasons (see online supplementary table S1), reflecting that the clinic operates at full capacity on most days.

\section{Men who have sex with men}

The odds of MSM being diagnosed with urethral gonorrhoea in summer was 1.23 times higher (95\% CI 1.04 to 1.46 ) than in winter. There was no seasonal difference in diagnoses of NGU or HSV (table 1).

The odds of MSM reporting a higher number of male sexual partners in the three months preceding consultations in summer was 1.05 times higher $(95 \%$ CI 1.03 to 1.07$)$ than in winter (table 2); this seasonal pattern was similar after we included those individuals triaged out of the service (table 3). After adjusting for known risk factors, the odds of being diagnosed with urethral gonorrhoea remained higher in summer than in winter $(\mathrm{aOR}=1.21,95 \% \mathrm{CI} 1.01$ to 1.45$)$ (table 1$)$.

\section{Heterosexual men}

Urethral gonorrhoea was not common among MSW, and while urethral gonorrhoea positivity was higher in summer than in winter, this difference was not statistically significant. The odds of MSW being diagnosed with NGU in summer was 1.11 times higher (95\% CI 1.03 to 1.20$)$ than in winter. There was no seasonal difference in HSV diagnoses (table 1).

The odds of MSW reporting a higher number of female sexual partners in the three months preceding consultations in summer was 1.09 times higher (95\% CI 1.06 to 1.12 ) than in winter (table 2); this seasonal pattern was similar after we included those individuals triaged out of the service (table 3 ). After adjusting for known risk factors, NGU diagnoses were no longer higher in summer than in winter (table 1).

\section{Women}

The proportion of women diagnosed with PID peaked in autumn, when the odds of being diagnosed with PID was 1.30 times higher (95\% CI 1.09 to 1.55$)$ than in winter. There was no seasonal difference in HSV diagnoses (table 1).

The odds of women reporting a higher number of male sexual partners in the three months preceding consultations in summer was 1.16 times higher (95\% CI 1.11 to 1.22 ) than in winter (table 2); this seasonal pattern was similar after we included those individuals triaged out of the service (table 3 ). After adjusting for known risk factors, PID diagnoses remained higher in autumn than in winter $(\mathrm{aOR}=1.29 ; 95 \% \mathrm{CI} 1.07$ to 1.54) (table 1).

Missing data are summarised in online supplementary table S1. 

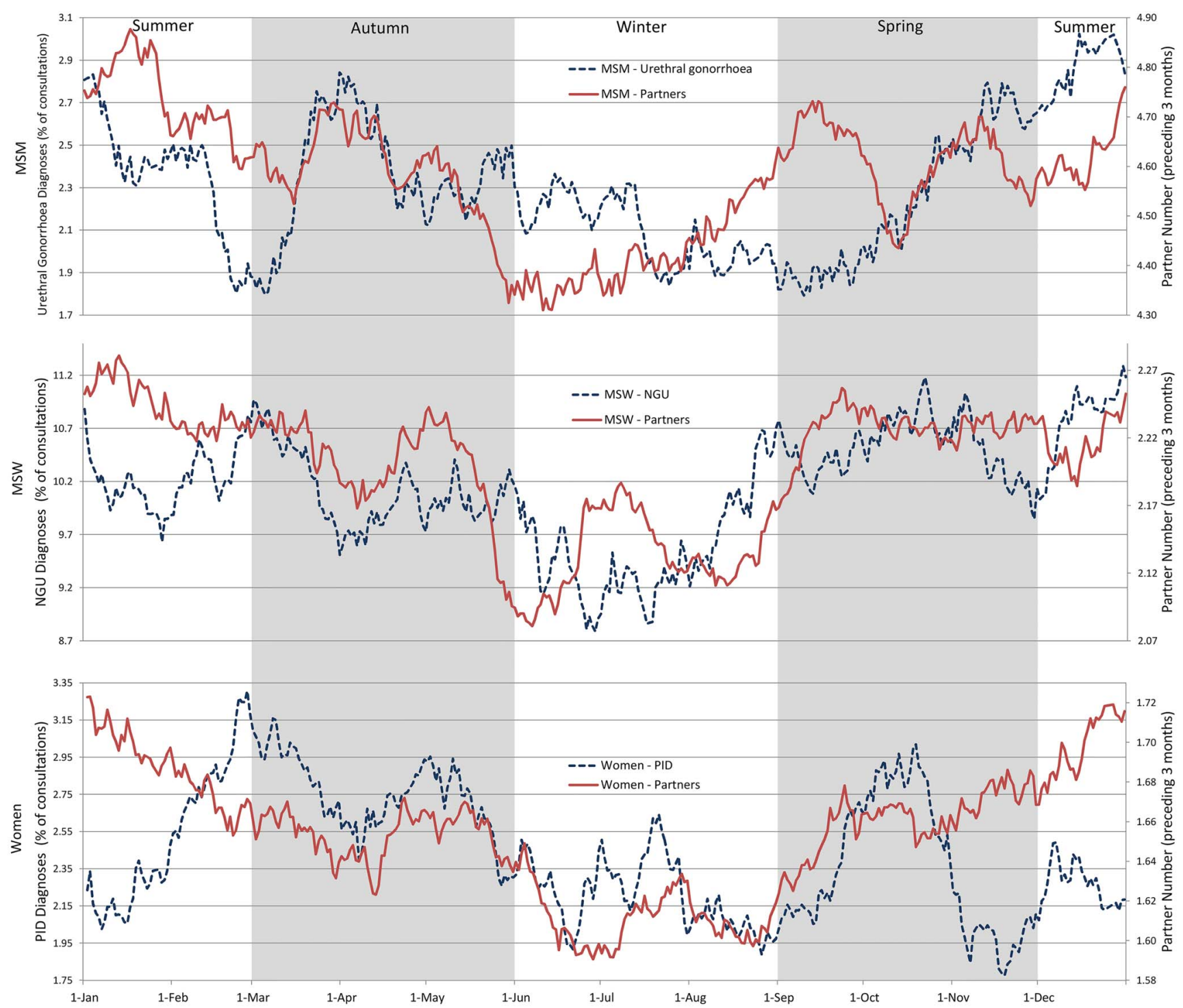

Figure 1 Daily 30-day centred moving averages of partner numbers and sexually transmitted infection (STI) diagnoses for men who have sex with men (MSM), men who have sex with women (MSW) and women. Y-axes do not start at zero. NGU, non-gonococcal urethritis; PID, pelvic inflammatory disease.

\section{DISCUSSION}

Our study demonstrates a small seasonal pattern in partner numbers for women, heterosexual men and MSM, with higher partner numbers reported for the preceding three months for consultations in summer compared with those in winter. This was accompanied by a small increase in some STI diagnoses in summer, though this seasonal difference was limited to urethral gonorrhoea in MSM and NGU in heterosexual men; PID diagnoses in women were found to peak in autumn. Previous studies have described either changes in sexual risk behaviour by season or changes in STI diagnoses by season, but not both. This is the first study to report both, and as a result, we were able to assess correlations between seasonal patterns of STI diagnoses and sexual risk behaviours to try to determine to what extent seasonal changes in STI diagnoses can be explained by changes in sexual risk behaviours.

There are two possible explanations for the seasonal difference in partner numbers: (1) changes in biological drive, that is, libido; and (2) changes in social opportunity for sex, such as holidays. The effect of social opportunity on sexual activity has been studied previously. A local 2014 analysis ${ }^{11}$ showed an overall higher number of presentations (not consultations, as some patients were triaged out due to service capacity constraints) at the MSHC in the months January to May compared with the rest of the year. They reported an increase in presentations during school vacation, 5 days after public holidays or university holidays and 5 days after major festivals in Melbourne, thus suggesting that social opportunity is an important factor. In the southern hemisphere, it is difficult to tease out the relative contribution of climactic effects and the contribution of social factors as the summer holidays include the Christmas period. As described in our introduction, some data from the northern hemisphere support the role of social constructs rather than biological determinants as a cause for seasonal differences in sexual behaviour, with peaks in condom sales over Christmas holidays and over summer holidays, ${ }^{5}$ and a similar annual pattern in gonorrhoea diagnoses. ${ }^{4}$ However, this pattern is not consistent across the literature as several retrospective analyses conducted in the USA have found a peak in STI rates in late summer and early autumn without a second peak after Christmas. ${ }^{1-3}$ It has 
Table 1 Seasonal sexually transmitted infection (STI) diagnoses in men who have sex with men (MSM), men who have sex with women (MSW) and women

\begin{tabular}{|c|c|c|c|c|c|c|}
\hline & & & Spring & Summer & Autumn & Winter \\
\hline \multirow[t]{12}{*}{ MSM } & Gonorrhoea & $\mathrm{N}$ & 11726 & 11393 & 11397 & 11736 \\
\hline & & $\mathrm{n}(\%)$ & $263(2.24 \%)$ & $293(2.57 \%)$ & $270(2.37 \%)$ & $246(2.10 \%)$ \\
\hline & & OR $(95 \% \mathrm{Cl})$ & $1.07(0.90$ to 1.28$)$ & $1.23^{*}$ (1.04 to 1.46$)$ & $1.13(0.95$ to 1.35$)$ & 1 (ref) \\
\hline & & aOR $(95 \% \mathrm{Cl}) \dagger$ & $1.06(0.89$ to 1.28$)$ & $1.21^{*}(1.01$ to 1.45$)$ & $1.14(0.95$ to 1.37$)$ & 1 (ref) \\
\hline & NGU & $\mathrm{N}$ & 11726 & 11393 & 11397 & 11736 \\
\hline & & $\mathrm{n}(\%)$ & $751(6.40 \%)$ & $733(6.43 \%)$ & $728(6.39 \%)$ & $788(6.71 \%)$ \\
\hline & & OR $(95 \% \mathrm{Cl})$ & $0.95(0.86$ to 1.05$)$ & $0.96(0.86$ to 1.06$)$ & $0.95(0.85$ to 1.05$)$ & 1 (ref) \\
\hline & & aOR $(95 \% \mathrm{Cl}) \dagger$ & 0.95 (0.85 to 1.06$)$ & $0.93(0.84$ to 1.06$)$ & $0.94(0.84$ to 1.05$)$ & 1 (ref) \\
\hline & HSV & $\mathrm{N}$ & 11726 & 11393 & 11397 & 11736 \\
\hline & & $\mathrm{n}(\%)$ & $99(0.84 \%)$ & $97(0.85 \%)$ & $102(0.90 \%)$ & $117(1.00 \%)$ \\
\hline & & OR $(95 \% \mathrm{Cl})$ & $0.85(0.65$ to 1.11$)$ & $0.85(0.65$ to 1.12$)$ & $0.90(0.69$ to 1.17$)$ & 1 (ref) \\
\hline & & aOR $(95 \% \mathrm{Cl}) \dagger$ & $0.88(0.66$ to 1.16$)$ & $0.86(0.64$ to 1.15$)$ & $0.89(0.67$ to 1.19$)$ & 1 (ref) \\
\hline \multirow[t]{12}{*}{ MSW } & Gonorrhoea & $\mathrm{N}$ & 13800 & 14035 & 14186 & 13821 \\
\hline & & $\mathrm{n}(\%)$ & $101(0.73 \%)$ & $105(0.75 \%)$ & $96(0.68 \%)$ & $80(0.58 \%)$ \\
\hline & & OR $(95 \% \mathrm{Cl})$ & $1.27(0.94$ to 1.70$)$ & $1.30(0.97$ to 1.73$)$ & $1.17(0.87$ to 1.58$)$ & 1 (ref) \\
\hline & & aOR $(95 \% \mathrm{Cl}) \dagger$ & $1.22(0.90$ to 1.65$)$ & 1.25 (0.93 to 1.69$)$ & $1.12(0.82$ to 1.52$)$ & 1 (ref) \\
\hline & NGU & $\mathrm{N}$ & 13800 & 14035 & 14186 & 13821 \\
\hline & & $\mathrm{n}(\%)$ & $1442(10.45 \%)$ & $1466(10.45 \%)$ & $1419(10.00 \%)$ & $1313(9.50 \%)$ \\
\hline & & OR $(95 \% \mathrm{Cl})$ & $1.11^{* *}(1.03$ to 1.20$)$ & $1.11^{* *}(1.03$ to 1.20$)$ & $1.06(0.98$ to 1.15$)$ & 1 (ref) \\
\hline & & aOR $(95 \% \mathrm{Cl}) \dagger$ & $1.07(0.98$ to 1.16$)$ & $1.07(0.99$ to 1.16$)$ & $1.03(0.95$ to 1.12$)$ & 1 (ref) \\
\hline & HSV & $\mathrm{N}$ & 13800 & 14035 & 14186 & 13821 \\
\hline & & $\mathrm{n}(\%)$ & $235(1.70 \%)$ & $207(1.47 \%)$ & $203(1.43 \%)$ & $208(1.50 \%)$ \\
\hline & & OR $(95 \% \mathrm{CI})$ & $1.13(0.94$ to 1.37$)$ & $0.98(0.81$ to 1.19$)$ & $0.95(0.78$ to 1.15$)$ & 1 (ref) \\
\hline & & aOR $(95 \% \mathrm{Cl}) \dagger$ & $1.08(0.89$ to 1.31$)$ & $0.93(0.77$ to 1.14$)$ & $0.90(0.74$ to 1.10$)$ & 1 (ref) \\
\hline \multirow[t]{8}{*}{ Women } & PID & $\mathrm{N}$ & 9740 & 10355 & 10586 & 9621 \\
\hline & & $\mathrm{n}(\%)$ & $227(2.33 \%)$ & $258(2.49 \%)$ & $298(2.82 \%)$ & $210(2.18 \%)$ \\
\hline & & OR $(95 \% \mathrm{Cl})$ & 1.07 (0.88 to 1.29$)$ & $1.15(0.95$ to 1.38$)$ & $1.30 * *(1.09$ to 1.55$)$ & 1 (ref) \\
\hline & & aOR $(95 \% \mathrm{Cl}) \dagger$ & $1.06(0.88$ to 1.28$)$ & 1.13 (0.94 to 1.36$)$ & $1.29 * *(1.07$ to 1.54$)$ & 1 (ref) \\
\hline & HSV & $\mathrm{N}$ & 9740 & 10355 & 10586 & 9621 \\
\hline & & $\mathrm{n}(\%)$ & $211(2.17 \%)$ & $202(1.95 \%)$ & $191(1.80 \%)$ & $195(2.03 \%)$ \\
\hline & & OR $(95 \% \mathrm{Cl})$ & $1.07(0.88$ to 1.30$)$ & $0.96(0.79$ to 1.17$)$ & $0.89(0.73$ to 1.09$)$ & 1 (ref) \\
\hline & & aOR $(95 \% \mathrm{Cl}) \dagger$ & $1.08(0.89$ to 1.32$)$ & $0.94(0.77$ to 1.15$)$ & $0.87(0.71$ to 1.07$)$ & 1 (ref) \\
\hline
\end{tabular}

ORs were adjusted for partner number over the last three months, condom use (always vs not always), age and year of consultation. Denominator ( $\mathrm{N}$ ) is the total number of consultations for each population. The numerator (n) represents the number of cases diagnosed, STI positivity in each season is expressed as per cent (\%), where the number of cases (n) divided by the total number of consultations $(\mathrm{N})$.

$p$ Values for OR and aOR: $p<0.05^{*}, p<0.01^{* *}, p<0.001^{* * *}$.

†Adjusted for number of partners and condom use in the past three months, age at consultation and year of consultation.

HSV, herpes simplex virus; NGU, non-gonococcal urethritis; PID, pelvic inflammatory disease.

Table 2 Seasonal behavioural risks (not including individuals triaged out)

\begin{tabular}{|c|c|c|c|c|c|c|}
\hline & & & Spring & Summer & Autumn & Winter \\
\hline MSM & $\begin{array}{l}\text { Partner numbert } \\
\text { Condom use (anal sex) } \ddagger \\
\text { Injecting drug use } \ddagger\end{array}$ & $\begin{array}{l}\text { Mean }( \pm \text { SD) } \\
\text { OR }(95 \% \mathrm{Cl}) \\
\% \\
\text { OR }(95 \% \mathrm{Cl}) \\
\% \\
\text { OR }(95 \% \mathrm{Cl})\end{array}$ & $\begin{array}{l}4.61( \pm 4.93) \\
1.02(1.00 \text { to } 1.04) \\
48.5 \% \\
0.97(0.92 \text { to } 1.03) \\
1.75 \% \\
1.13(0.92 \text { to } 1.38)\end{array}$ & $\begin{array}{l}4.71( \pm 4.95) \\
1.05^{* * *}(1.03 \text { to } 1.07) \\
48.9 \% \\
0.99(0.93 \text { to } 1.04) \\
1.21 \% \\
0.78^{*}(0.62 \text { to } 0.97)\end{array}$ & $\begin{array}{l}4.57( \pm 4.88) \\
1.02(1.00 \text { to } 1.04) \\
49.3 \% \\
1.00(0.95 \text { to } 1.06) \\
1.72 \% \\
1.11(0.90 \text { to } 1.36)\end{array}$ & $\begin{array}{l}4.42( \pm 4.68) \\
1 \text { (ref) } \\
49.2 \% \\
1 \text { (ref) } \\
1.55 \% \\
1 \text { (ref) }\end{array}$ \\
\hline MSW & $\begin{array}{l}\text { Partner numbert } \\
\text { Condom use } \\
\text { (vaginal or anal sex)‡ } \\
\text { Injecting drug use }\end{array}$ & $\begin{array}{l}\text { Mean }( \pm \mathrm{SD}) \\
\text { OR }(95 \% \mathrm{Cl}) \\
\% \\
\text { OR }(95 \% \mathrm{Cl}) \\
\% \\
\text { OR }(95 \% \mathrm{Cl})\end{array}$ & $\begin{array}{l}2.46( \pm 2.67) \\
1.07^{* * *}(1.04 \text { to } 1.10) \\
20.4 \% \\
0.92^{*}(0.87 \text { to } 0.98) \\
0.94 \\
0.94(0.74 \text { to } 1.20)\end{array}$ & $\begin{array}{l}2.46( \pm 2.48) \\
1.09^{* * *}(1.06 \text { to } 1.12) \\
20.5 \% \\
0.93^{*}(0.88 \text { to } 0.99) \\
0.75 \\
0.75^{*}(0.58 \text { to } 0.96)\end{array}$ & $\begin{array}{l}2.43( \pm 2.52) \\
1.06^{* * *}(1.03 \text { to } 1.09) \\
20.8 \% \\
0.94(0.89 \text { to } 1.01) \\
0.90 \\
0.90(0.71 \text { to } 1.15)\end{array}$ & $\begin{array}{l}2.31( \pm 2.51) \\
1(\mathrm{ref}) \\
21.7 \% \\
1 \text { (ref) } \\
1.00 \\
1 \text { (ref) }\end{array}$ \\
\hline Women & $\begin{array}{l}\text { Partner numbert } \\
\text { Condom use } \\
\text { (vaginal or anal sex)‡ } \\
\text { Injecting drug use } \ddagger\end{array}$ & $\begin{array}{l}\text { Mean }( \pm \mathrm{SD}) \\
\text { OR } 95 \% \mathrm{Cl} \\
\% \\
\text { OR }(95 \% \mathrm{Cl}) \\
\% \\
\text { OR }(95 \% \mathrm{Cl})\end{array}$ & $\begin{array}{l}1.77( \pm 1.47) \\
1.09^{* *}(1.03 \text { to } 1.14) \\
17.4 \% \\
0.98(0.91 \text { to } 1.06) \\
0.72 \% \\
1.10(0.78 \text { to } 1.55)\end{array}$ & $\begin{array}{l}1.83( \pm 1.64) \\
1.16^{\star * *}(1.11 \text { to } 1.22) \\
17.6 \% \\
0.99(0.92 \text { to } 1.07) \\
0.66 \% \\
1.01(0.71 \text { to } 1.43)\end{array}$ & $\begin{array}{l}1.76( \pm 1.37) \\
1.08^{* *}(1.02 \text { to } 1.13) \\
18.2 \% \\
1.04(0.97 \text { to } 1.12) \\
0.69 \% \\
1.06(0.75 \text { to } 1.49)\end{array}$ & $\begin{array}{l}1.72( \pm 1.72) \\
1(\mathrm{ref}) \\
17.7 \% \\
1(\mathrm{ref}) \\
0.65 \% \\
1 \text { (ref) }\end{array}$ \\
\hline
\end{tabular}

Data on partner numbers, condom use and injecting drug use are that reported for the three months preceding the consultation.

$\mathrm{p}$ Values for $\mathrm{OR}: \mathrm{p}<0.05^{*}, \mathrm{p}<0.01 * *, \mathrm{p}<0.001 * * *$.

TThe reported mean partner numbers are derived from the raw data, the ORs are derived from recoded data, as described in the 'Methods' section.

¥Condom use data and injecting drug use data is binary, being 'always' versus 'not always' for condom use, and 'yes' versus 'no' for injecting drug use.

MSM, men who have sex with men; MSW, men who have sex with women. 
Table 3 Mean partner numbers, including people triaged out, by season

\begin{tabular}{|c|c|c|c|c|c|}
\hline & & Spring & Summer & Autumn & Winter \\
\hline \multirow[t]{2}{*}{ MSM } & Mean $( \pm S D)$ & $5.29( \pm 8.33)$ & $5.54( \pm 9.36)$ & $5.29( \pm 8.66)$ & $5.13( \pm 8.32)$ \\
\hline & OR $(95 \% \mathrm{Cl})$ & 1.02 (0.997 to 1.04$)$ & $1.05^{* * *}$ (1.03 to 1.08$)$ & 1.02 (0.999 to 1.04$)$ & 1 (ref) \\
\hline \multirow[t]{2}{*}{ MSW } & Mean $( \pm S D)$ & $2.46( \pm 2.67)$ & $2.46( \pm 2.48)$ & $2.43( \pm 2.52)$ & $2.31( \pm 2.51)$ \\
\hline & OR $(95 \% \mathrm{Cl})$ & $1.07^{* * *}(1.04$ to 1.10$)$ & $1.09^{* * *}(1.06$ to 1.12$)$ & $1.06^{* * *}(1.03$ to 1.09$)$ & 1 (ref) \\
\hline \multirow[t]{2}{*}{ Women } & Mean $( \pm S D)$ & $1.72( \pm 2.07)$ & $1.81( \pm 2.73)$ & $1.69( \pm 1.61)$ & $1.64( \pm 1.79)$ \\
\hline & OR $(95 \% \mathrm{Cl})$ & $1.12^{* * *}(1.06$ to 1.16$)$ & $1.20^{* * *}(1.15$ to 1.25$)$ & $1.08^{* * *}(1.04$ to 1.13$)$ & 1 (ref) \\
\hline
\end{tabular}

$p$ Values for OR: $p<0.05^{*}, p<0.01^{* *}, p<0.001^{* * *}$.

MSM, men who have sex with men; MSW, men who have sex with women.

been suggested that the discrepancy in seasonal data between different studies is due to societal changes over time, so that older studies are influenced mainly by biological changes in sexual drive in a socially conservative environment, whereas newer studies describe annual patterns of sexual behaviour that result from social opportunity for sex during specific annual events. $^{12}$

Our data support previous findings of seasonal differences in partner numbers and seasonal differences in STI diagnoses, and we were able to assess the contribution of seasonal differences in sexual risk behaviours to the observed seasonal differences in STI diagnoses. We found that in MSM the seasonal difference in urethral gonorrhoea diagnoses persisted after we adjusted for partner numbers, condom use and IDU. This suggests that other factors must contribute to the rise in urethral gonorrhoea diagnoses in summer. A possible explanation is provided by the authors of a recent analysis ${ }^{13}$ of chlamydia and gonorrhoea diagnoses in MSM at the MSHC. They reported that diagnoses of chlamydia and gonorrhoea increased substantially between 2007 and 2013, but that at the same time there was a substantial reduction in number of sexual partners and only a minimal decrease in condom use. A further analysis of this apparent contradiction showed that the increase in STI diagnoses was restricted to those individuals with a high number of sexual partners, causing the authors to hypothesise that the rise in STI diagnoses is a result of changes in partner mixing patterns, with high-risk partners now mixing together more commonly, thereby amplifying their STI risk beyond that expected for their partner number. Such amplification through altered partner mixing would also explain our seasonal findings. An alternative explanation for the persistence of seasonal difference in urethral gonorrhoea diagnoses in MSM after adjusting for partner numbers is that the frequent use of antibiotics for respiratory infections in winter may treat asymptomatic gonorrhoea infections of the throat and rectum, ${ }^{2}$ thereby reducing community reservoirs and potentially reducing urethral gonorrhoea incidence. This hypothesis is supported by the observation of increased antibiotic resistance in gonococcal cultures in winter. $^{14}$

When we adjust for known risk factors, there is no longer a seasonal difference in NGU diagnoses in MSW. This suggests that the cause of the rise in NGU is a rise in partners numbers but also suggests that some of the factors that underlie the seasonal differences in urethral gonorrhoea diagnoses among MSM do not apply to NGU diagnoses among MSW.

There are several factors that need to be considered when interpreting our findings. First, there is an inherent problem in the data set, in that the STI data can accurately be assigned to a season according to the date of testing, but the data on partner numbers are reported for the 3 -month period preceding the date of testing. Hence, patients who present at the start of the season are likely to be reporting partners with whom they had contact in the previous season. Second, this study is a retrospective analysis of records, limited to a single urban sexual health clinic; our findings may not be generalisable to other settings. Third, it is possible that our findings do not reflect a general rise in STI risk in the community, but rather that our service experiences greater demand in summer and hence the triage system selects only the highest risk clients for assessment during the summer period. We consider this unlikely because the sexual risk factors for all clients (including those triaged out) rose during summer, and the seasonal risk pattern was similar for those individuals triaged out as it was for those individuals who received clinical assessment.

Our findings may have public health implications: public health messages promoting sexual safety are likely to have a greater impact in spring and summer rather than in winter; perhaps health promotion campaigns should intensify during these times of year. In particular, it is likely that such messages have maximal impact during annual events during which people are at increased risk, for example, on Valentine's day or during Sydney's Mardi Gras and Melbourne's Midsumma Carnival. Previous work has shown that people generally have a preference for receiving sexual health promotion messages during times of increased risk. ${ }^{15}$

Further research is needed to clarify how the seasonal pattern in partner numbers and STIs may be different for people in different age groups, and what other risk factors underlie this seasonal difference. In particular, further work is needed to clarify the factors underlying the seasonal differences in urethral gonorrhoea rates among MSM, which may then help to inform health promotion strategies.

\section{Key messages}

- Previous studies have assessed either seasonal differences in partner number or seasonal differences in sexually transmitted infection (STI) diagnoses. This is the first study to assess both.

- In Melbourne, Australia, people report higher numbers of sexual partners in the three months before consultations in summer compared with winter.

- STI diagnoses in men are highest in summer.

- Among men who have sex with men (MSM), the peak in diagnoses in summer is larger than expected by increases in partner number. This suggests other factors such as altered mixing patterns by season among MSM.

\section{Handling editor Jackie A Cassell}

Acknowledgements The authors acknowledge A Afrizal for his assistance with data extraction. 
Contributors EPFC and CKF conceived the study. VJC and EPFC undertook the analysis. VJC prepared the first draft and all authors contributed to and approved of the final version.

Funding This work was supported by the National Health and Medical Research Council (NHMRC) programme grant (no: 568971).

Competing interests EPFC is supported by the Early Career Fellowships from the Australian NHMRC (no: 1091226).

Ethics approval Ethics Committee, The Alfred Hospital, Melbourne, Australia (approval number 42/15).

Provenance and peer review Not commissioned; externally peer reviewed.

\section{REFERENCES}

1 Cornelius CE III. Seasonality of gonorrhea in the United States. HSMHA Health Rep 1971;86:157-60.

2 Wright RA, Judson FN. Relative and seasonal incidences of the sexually transmitted diseases. A two-year statistical review. Br J Vener Dis 1978;54:433-40.

3 Schroeder B, Tetlow P, Sanfilippo JS, et al. Is there a seasonal variation in gonorrhea and chlamydia in adolescents? J Pediatr Adolesc Gynecol 2001;14:25-7.

4 Ross JD, Scott GR. Seasonal variation in gonorrhoea. Eur J Epidemiol 1992; $8: 252-5$

5 Wellings K, Macdowall W, Catchpole M, et al. Seasonal variations in sexual activity and their implications for sexual health promotion. J R Soc Med 1999;92:60-4.

6 Damiba AE, Vermund SH, Kelley KF. Rising trend of reported gonorrhoea and urethritis incidence in Burkina Faso from 1978 to 1983. Trans R Soc Trop Med Hyg 1990;84:132-5.
7 Rodgers JL, Harris DF, Vickers KB. Seasonality of first coitus in the United States. Soc Biol 1992;39:1-14.

8 Fortenberry JD, Orr DP, Zimet GD, et al. Weekly and seasonal variation in sexual behaviors among adolescent women with sexually transmitted diseases. J Adolesc Health 1997;20:420-5.

9 Chow EP, Fehler G, Chen MY, et al. Testing commercial sex workers for sexually transmitted infections in Victoria, Australia: an evaluation of the impact of reducing the frequency of testing. PLOS ONE 2014;9:e103081.

10 Vodstrcil LA, Hocking JS, Cummings R, et al. Computer assisted self interviewing in a sexual health clinic as part of routine clinical care; impact on service and patient and clinician views. PLOS ONE 2011;6:e18456.

11 Gamagedara N, Hocking JS, Law $\mathrm{M}$, et al. What are seasonal and meteorological factors are associated with the number of attendees at a sexual health service? An observational study between 2002-2012. Sex Transm Infect 2014;90:635-40.

12 Shah AP, Smolensky MH, Burau KD, et al. Recent change in the annual pattern of sexually transmitted diseases in the United States. Chronobiol Int 2007;24:947-60.

13 Chow EP, Tomnay J, Fehler G, et al. Substantial increases in chlamydia and gonorrhea positivity unexplained by changes in individual-level sexual behaviors among men who have sex with men in an Australian sexual health service from 2007 to 2013. Sex Transm Dis 2015;42:81-7.

14 Jaffe HW, Zaidi AA, Thornsberry C, et al. Trends and seasonality of antibiotic resistance of Neisseria gonorrhoeae. J Infect Dis 1977;136:684-8.

15 Gold J, Lim MS, Hellard ME, et al. What's in a message? Delivering sexual health promotion to young people in Australia via text messaging. BMC Public Health 2010;10:792 